\title{
Screening e trattamento delle neoplasie nel follow-up del trapiantato renale
}

\author{
M. Zanazzi, A. Larti, L. Di Maria, P. Carta, G. Rosso, E. Bertoni
}

Nefrologia dei Trapianti, Azienda Ospedaliera Universitaria Careggi, Firenze

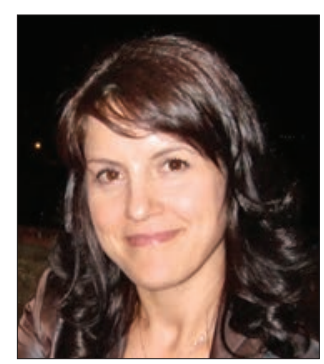

Aida Larti

\section{Introduzione}

La disponibilità di nuovi farmaci immunosoppressori e l'avanzamento delle tecnologie hanno consentito un netto miglioramento dei risultati a breve e a lungo termine nel trapianto renale. Tuttavia, nonostante i progressi realizzati, le problematiche nella fase del post-trapianto risultano numerose e tra le più difficili da affrontare dal punto di vista clinico. Le malattie cardiovascolari sono la principale causa di morte del trapianto renale, ma le neoplasie rappresentano un altrettanto grave problema che inciderà sulla sopravvivenza a lungo termine dei pazienti nei prossimi vent'anni. I dati in letteratura dimostrano una incidenza da tre a cinque volte superiore di neoplasia nel trapiantato renale rispetto alla popolazione generale.

Negli ultimi trent'anni la sopravvivenza dei pazienti dopo trapianto renale è notevolmente migliorata (1). Attualmente, i tassi di sopravvivenza risultano del 95\% a 1 anno e di circa il 90\% dopo 5 anni (2); tuttavia, l'aspettativa di vita rimane di gran lunga peggiore rispetto alla popolazione generale (2). United States Renal Data System (USRDS) riporta una percentuale di decessi nel post-trapianto renale, attribuibili a neoplasia, pari al 7,5\%. Rispetto alla popolazione generale, i pazienti trapiantati renali sviluppano più frequentemente alcuni tipi di neoplasie che sono spesso più aggressive e offrono una prognosi peggiore (1). In uno studio recente, che ha valutato più di 2000 pazienti trapiantati renali da più di vent'anni, il 40\% circa dei pazienti ha sviluppato tumori cutanei e, circa il $10 \%$, un tumore solido (3).

Alcune neoplasie insorgono comunemente nella popolazione generale e risultano pure di frequente riscontro nei trapiantati renali, come il tumore del colon; altri insorgono nei trapiantati renali con una incidenza simile alla popolazione generale, come la neoplasia mammaria $(4,5)$. Altre neoplasie rare nella popolazione generale risultano di sostanziale appannaggio della popolazione dei trapiantati, come il sarcoma di Kaposi, con una insorgenza di quaranta volte più frequente nel trapiantato renale rispetto alla popolazione generale (2).

Dopo la comparsa della neoplasia, la sopravvivenza del paziente trapiantato risulta compromessa con limitate possibilità di intervento per la presenza appunto del trapianto e di fattori di comorbidità.

E appunto per questa ragione che risulta essenziale porre in campo misure di screening, atte ad identificare precocemente l'insorgenza delle neoplasie ed affrontare con idonee misure l'evoluzione della patologia.

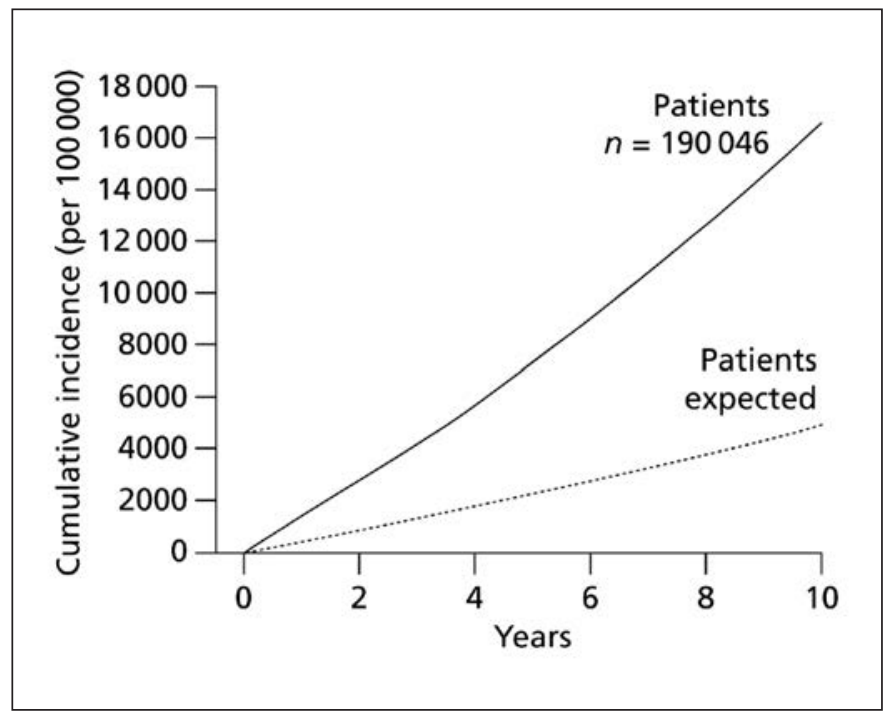

Fig. 1 - Incidenza di tutti i tipi di tumore maligno nei pazienti riceventi un trapianto di rene da cadavere: confronto con l'incidenza attesa nella popolazione generale. CTS K51109-0206. 


\section{Screening delle neoplasie nel paziente trapiantato renale}

La finalità dello screening delle neoplasie nel paziente trapiantato renale è quella di identificare precocemente la lesione, di poter intervenire in modo immediato con le diverse armi terapeutiche (terapia medica, chirurgica, variazione del trattamento immunosoppressivo), con un'azione che può risultare sostanzialmente differente rispetto alla popolazione generale. I pazienti trapiantati presentano numerose comorbidità che possono influenzare i benefici di un precoce screening, soprattutto per i tumori solidi: è chiaro che la decisione dello screening per tumore deve essere sicuramente individualizzata, in relazione all'anamnesi familiare, alle abitudini di vita e alla storia clinica (fumo di sigaretta, esposizione al sole, abuso di analgesici, storia di neoplasia precedente al trapianto, età del paziente, sesso, regione geografica di provenienza, razza, predisposizione genetica, presenza di virus oncogeni) .

Lo screening di alcuni tumori fa riferimento sostanzialmente alle linee guida individuate per la popolazione generale.

Per il sesso femminile: il tumore della cervice uterina, della mammella e del colon. Per il sesso maschile: il tumore della prostata e del colon.

\section{Screening del tumore della cervice uterina}

I benefici dello screening nella popolazione generale sono universalmente riconosciuti. Nella trapiantata renale il tumore della cervice uterina è estremamente comune, per cui lo screening risulta indispensabile. Nella popolazione generale esiste una correlazione con il papilloma virus (HP) e quindi è stata suggerita la vaccinazione. Nelle trapiantate la vaccinazione risulta meno efficace. In queste pazienti il tumore della cervice uterina può evolvere rapidamente e risultare decisamente aggressivo, probabilmente legato al trattamento immunosoppressivo, tanto che nelle trapiantate renali lo screening deve essere eseguito più frequentemente. Le linee guida europee e americane del trapianto renale suggeriscono uno screening con cadenza annuale. Lo screening permette inoltre una valutazione della regione anale, vaginale e vulvare, sedi che possono essere frequentemente colpite da neoplasie nelle pazienti trapiantate. La vaccinazione per HP nelle giovani donne è stata adottata in diverse nazioni. Il vaccino è un vaccino inattivato e può essere utilizzato sia prima del trapianto che nella paziente trapiantata, anche se non è stata dimostrata sicura efficacia e sicurezza nei pazienti immunosoppressi.
Alcune Società internazionali attualmente raccomandano l'utilizzazione del HPV DNA in aggiunta allo screening istologico.

\section{Screening del tumore della mammella}

Sia nella popolazione generale che nelle pazienti trapiantate lo screening per il tumore mammario risulta un evento consolidato. Risulta ormai assodato che la mammografia utilizzata nello screening nelle donne della popolazione generale di età compresa fra 50-74 anni riduce la mortalità del tumore del $23 \%$. Lincidenza del tumore della mammella risulta simile in entrambe le popolazioni. Anche le linee guida americane raccomandano lo screening con la mammografia nelle donne fra 5069 anni e ponendo una opzione di valutazione al Centro Senologico di riferimento per le donne di età superiore a 40 anni. L'accuratezza della mammografia risulta maggiore nelle donne più anziane con risultati peggiori nelle donne più giovani.

\section{Screening del tumore della prostata}

L'utilizzo dell'antigene prostatico specifico (PSA) e/o della esplorazione rettale nella popolazione generale come screening per il carcinoma della prostata risulta ancora controverso. Le più recenti raccomandazioni identificano come limite allo screening un'età superiore ai 75 anni. L'incidenza delle neoplasie nei pazienti trapiantati è simile a quella della popolazione generale. Risulta comunque importante considerare che il carcinoma della prostata rappresenta uno dei tumori più frequenti nel sesso maschile e si deve quindi programmare un attento follow-up.

\section{Screening del tumore del colon}

Anche per quanto riguarda lo screening del tumore del colon nella popolazione generale, ci sono buone evidenze che dimostrano il beneficio dello screening nelle persone di età maggiore di 50 anni. Ovviamente l'incidenza del tumore del colon è superiore nelle persone trapiantate di età maggiore ai 50 anni rispetto alla popolazione generale della stessa età. La ricerca del sangue occulto nelle feci nella popolazione dei trapiantati sembra sia più specifica che nella popolazione generale, ma non ci sono evidenze che dimostrano che la colonscopia sia meno sensibile o meno specifica.

Le linee guida australiane, americane ed europee raccomandano lo screening della popolazione generale con la ricerca del sangue occulto nelle feci e/o colonscopia nella popolazione con età maggiore di 50 anni. Per 
quanto riguarda le linee guida delle Società americane ed europee dei trapianti, viene sempre raccomandato lo screening nella popolazione dei trapiantati con età maggiore di 50 anni.

Lo screening con la ricerca del sangue occulto nelle feci può essere meno specifico nella popolazione dei trapiantati considerando l'alta incidenza dei falsi positivi legati all'infezione da CMV o alla tossicità dei farmaci immunosoppressivi.

Una recente analisi ha però dimostrato che possono essere riscontrati benefici maggiori se lo screening viene effettuato nella popolazione dei trapiantati con età compresa fra 35 e 50 anni.

\section{Screening del tumore del fegato}

Nella popolazione dei trapiantati, il rischio del carcinoma epatocellulare è più alto rispetto a quello della popolazione generale. Nella popolazione generale non ci sono chiare evidenze di benefici portati dall'effettuazione dello screening.

Vi sono però raccomandazioni di eseguire uno screening appropriato nella popolazione ad alto rischio come pazienti affetti da cirrosi epatica o portatori di virus dell'epatite B. Tale screening prevede l'esecuzione di una ecografia addominale ed il dosaggio di alpha-feto-proteina ogni 6-12 mesi. Questi esami hanno dimostrato comunque una bassa sensibilità e specificità.

\section{Screening del tumore dei reni nativi}

I carcinomi renali rappresentano una delle neoplasie a più comune insorgenza nel paziente trapiantato e nel rene assume particolare importanza la presenza della malattia cistica acquisita (ACKD). L'ACKD è stata descritta come la comparsa di formazioni cistiche in un rene terminale, precedentemente non cistico e la comparsa di malattia può essere associata alla durata del trattamento dialitico. L'ACKD rappresenta infatti un fattore di rischio ben conosciuto per l'insorgenza di neoplasia renale.

L'ultrasonografia è un metodo accurato e minimamente invasivo utile nella diagnosi e nel follow-up delle lesioni solide e cistiche del rene, ma presenta una sensibilità limitata nei confronti delle masse di piccole dimensioni e delle formazioni cistiche complesse. La ACKD rappresenta una patologia da seguire nel tempo per la sua alta probabilità di degenerare in un carcinoma renale; non esiste tuttavia un consenso unanime da parte della comunità scientifica internazionale per la tempistica e le modalità di esecuzione del follow-up. Le linee guida americane ed europee raccomandano in prima istanza un follow-up ecografico e successivamente una TC con mezzo di contrasto iodato qualora si riscontrino formazioni dubbie all'ecografia, ma non esistono specifiche indicazioni sui tempi di acquisizione di questi esami. Negli ultimi dieci anni lo sviluppo di mezzi di contrasto ecografici ha permesso lo studio, da parte dell'ultrasonografia, della microcircolazione dei parenchimi. L'introduzione di tale metodica risale ai primi anni Novanta, ma l'entrata in commercio di mezzi di contrasto di seconda generazione ha permesso un notevolissimo passo in avanti nello studio non invasivo di patologie di vario genere in particolare della vascolarizzazione delle masse solide epatiche e successivamente renali.

\section{L'impatto del trattamento immunosoppressivo}

La terapia immunosoppressiva, così importante per il successo del trapianto, rappresenta anche una delle principali cause di morbilità e mortalità del paziente trapiantato. La malattia cardiovascolare, le infezioni e la patologia neoplastica, che risultano le principali cause di mortalità del paziente trapiantato, sono infatti in gran parte favorite dai farmaci immunosoppressori di per sé o dallo stato di alterata sorveglianza immunologica che deriva dalla loro somministrazione. La relazione tra la natura, l'intensità, la durata della terapia immunosoppressiva e l'insorgenza della neoplasia è ormai ben definita (6), anche se l'uso di terapie combinate rende difficile valutare l'impatto dei singoli agenti immunosoppressivi sul rischio neoplastico. I farmaci antilinfocitari, come le globuline antilinfociti ed antitimociti, favoriscono indubbiamente l'insorgenza delle neoplasie, ma tale effetto non sembra ascrivibile ai farmaci di per sé, ma alla gravità dell'immunosoppressione indotta. A parte gli inibitori del segnale di proliferazione (mTOR), Sirolimus ed Everolimus, tutti gli altri farmaci immunosoppressori utilizzati possono essere ugualmente ed indifferentemente considerati fattori di rischio per l'insorgenza delle neoplasie. È stata ben documentata, sia in trials clinici che da singoli reports (7), l'efficacia degli inibitori di mTOR nel prevenire diversi tipi di tumore nei pazienti trapiantati o nel permettere la regressione di tumori che si sono sviluppati. In uno studio di Campistol et al i pazienti in terapia con Sirolimus sviluppavano meno tumori di quelli in terapia con inibitori della calcineurina (8). Come pure è stata documentata una completa guarigione del sarcoma di Kaposi dopo con- 


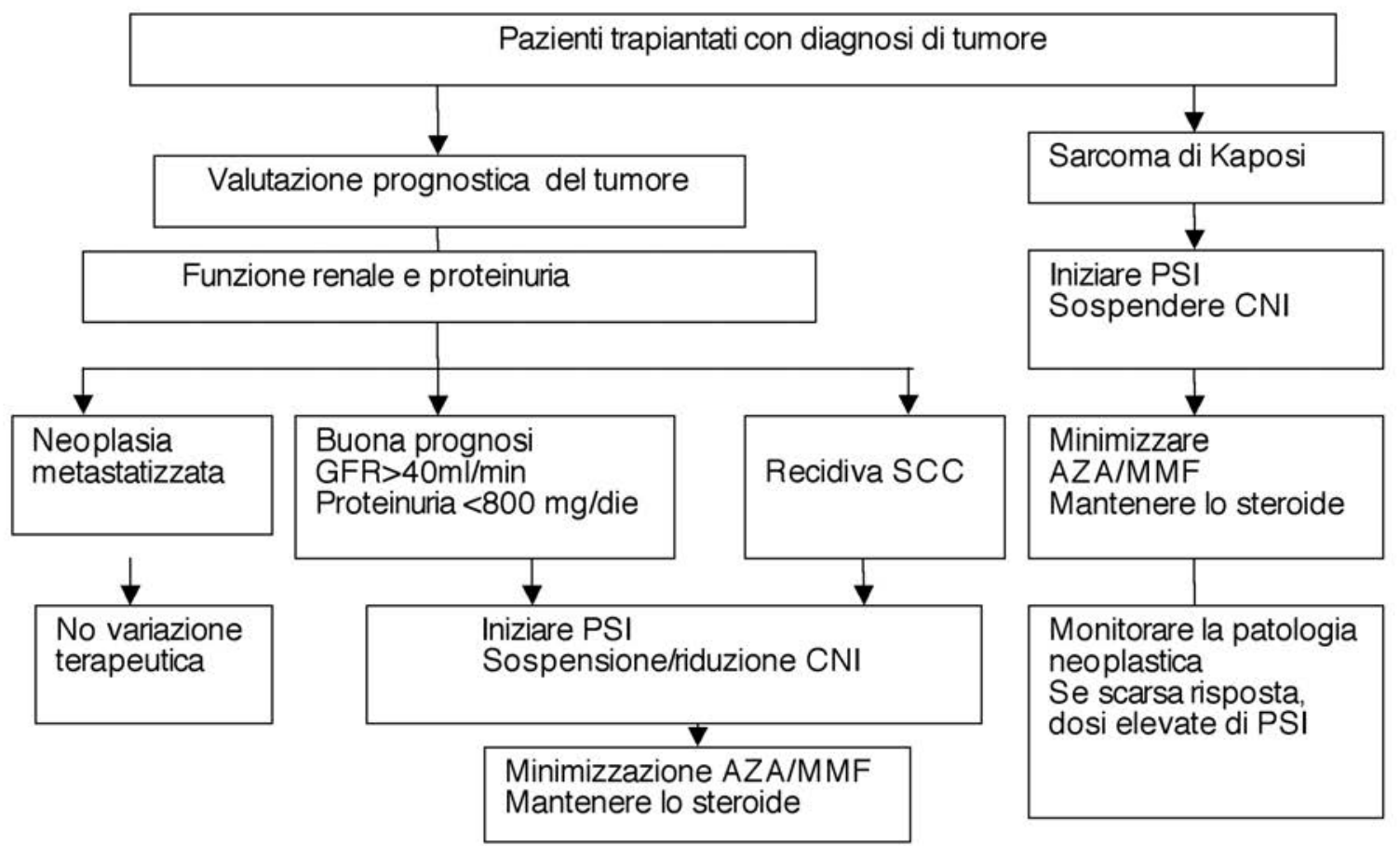

Fig. 2 - Tradotto da Campistol JM et al. Nephrol Dial Transplant 2007; 22 Suppl 1: i36-41.

versione da inibitori della calcineurina a terapia con Sirolimus (9). Lo switch è solitamente ben tollerato con funzione renale preservata ed assenza di rigetto nella maggior parte dei pazienti.

Gli inibitori di mTOR sembrano pertanto indicati per tutti quei pazienti che hanno presentato una neoplasia dopo il trapianto renale, tenendo presente alcune fondamentali raccomandazioni da seguire al momento dell'eventuale conversione da inibitori della calcineurina agli inibitori del segnale di proliferazione (PSI) (Fig. 2) (10). In considerazione del ruolo espresso dalla terapia immunosoppressiva nel causare o aggravare diversi tipi di neoplasia, l'orientamento generale è quello di ridurre il carico complessivo dei farmaci, ma ridurre la terapia immunosoppressiva nel paziente trapiantato non è una facile decisione da prendere, pur nella consapevolezza che la riduzione possa in qualche modo prolungare la sopravvivenza e ridurre le complicanze che insorgono in concomitanza dei trattamenti chemioterapici. Un altro elemento da sottolineare è la possibilità che la riduzione del trattamento immunosoppressivo possa aumentare il rischio di rigetto acuto e con esso una maggior probabilità di perdita del graft, con un forte impatto sulla qualità di vita del paziente. Risulta pertanto indispensabile, a nostro parere, la costituzione di una équipe multidisciplinare (nefrologo, oncologo, radiologo), per la scelta della migliore opzione terapeutica, adeguata alle caratteristiche del paziente, valutandone l'età, il tipo di neoplasia, lo stadio della malattia neoplastica, oltre alle specifiche richieste del paziente.

Indirizzo degli Autori:

Dott.ssa Maria Zanazzi

U. O. Nefrologia dei Trapianti e Dialisi

A.O.U. Careggi Firenze

Viale Pieraccini 17

50139 Firenze

maria.zanazzi@libero.it 


\section{Bibliografia}

1. Brigg JD. Causes of death after transplantation. Nefrol Dial Transplant 2001; 16: 1545-9.

2. ANZDATA. Australia and New Zealand Dyalisis and Transplant Registry. 2006 (accessed october 2006).avaible at:http:॥www.anzdada.org.au/.

3. Matas AJ, Gillingham KJ, Humar A, et al. Kidney transplant recipients with 10 years of graft function: what happens next? Am J Transplant 2008; 8: 2410-9.

4. Ulrich C, Uchmook T, Sachse MM, et al. Comparative epidemiology and pathogenic factors for nonmelanoma skin cancer in organ transplant patients. Dermatol Surg 2004; 30: 622-7.

5. Opelz G, Dohler B. Lymphomas after solid organ transplantation: a collaborative transplant study report. Am J transplant 2004; 4: 222-30.
6. Benett W, Vella JP. Transplantation. Nephsap 2005; 4: 483.

7. Salvadori M, Bertoni E. Sorveglianza immunologica ed oncogenesi: ruolo degli inibitori del segnale di proliferazione. G Ital Nefrol 2010; s10-s18.

8. Campistol JM, Eris J, Oberbauer R, et al. Sirolimus therapy after early cyclosporine withdrawal reduces the risk for cancer in adult renal transplantation. J Am Soc Neph 2006; 17: 581-9.

9. Stallone $G$, Infante $B$, Schena $A_{2}$ et al. Rapamycin for treatment of chronic allograft nephropathy in renal transplant patients. J Am Soc Nephrol_2005; 16: 3755-62.

10. Campistol JM, Albanell J, Arns W, et al. Use of proliferation signal inhibitors in the management of post-transplant malignancies-clinical guidance. Nephrol Dial Transplant 2007; 22 (Suppl 1): i36-41. 